\title{
ON THE $r$-TH HYPER-KLOOSTERMAN SUMS AND ITS HYBRID MEAN VALUE
}

\author{
TIANPING Zhang AND WENPENG ZHANG
}

\begin{abstract}
The main purpose of this paper is using the properties of Gauss sums, primitive characters and the mean value theorems of Dirichlet L-functions to study the hybrid mean value of the $r$-th hyper-Kloosterman sums $K l(h, k+1, r ; q)$ and the hyper Cochrane sums $C(h, q ; m, k)$, and give an interesting mean value formula.
\end{abstract}

\section{Introduction}

For any positive integer $q$ and $n$ and an arbitrary integer $h$, the general Dedekind sums $S(h, n, q)$ is defined by

$$
S(h, n, q)=\sum_{a=1}^{q} \bar{B}_{n}\left(\frac{a}{q}\right) \bar{B}_{n}\left(\frac{a h}{q}\right)
$$

where

$$
\bar{B}_{n}(x)= \begin{cases}B_{n}(x-[x]), & \text { if } x \text { is not an integer; } \\ 0, & \text { if } x \text { is an integer. }\end{cases}
$$

$B_{n}(x)$ is the Bernoulli polynomial, and $\bar{B}_{n}(x)$ is the $n$-th Bernoulli periodic function, defined on the interval $0<x \leq 1$. In [8] and [6], the second author has given some mean value properties of $S(h, n, q)$. In October 2000, during his visit to Xi'an, Professor Todd Cochrane introduced the follcwing sums analogous to the Dedekind sums,

$$
C(h, q)=\sum_{a=1}^{q}\left(\left(\frac{\bar{a}}{q}\right)\right)\left(\left(\frac{a h}{q}\right)\right),
$$

Received May 30, 2005. Revised November 12, 2005.

2000 Mathematics Subject Classification: 11N37, 11F20.

Key words and phrases: hyper-Kloosterman sums, hyper Cochrane sums, hybrid mean value.

This work was supported by the N.S.F.(10271093, 60472068) and P. N. S. F. of P. R. China. 
where $\sum^{\prime}$ denotes the summation over all $a$ such that $(a, q)=1, a \bar{a} \equiv 1$ $\bmod q$, and

$$
((x))= \begin{cases}x-[x]-\frac{1}{2} & \text { if } x \text { is not an integer } \\ 0 & \text { if } x \text { is an integer }\end{cases}
$$

He advised us to study the arithmetical properties and mean value distribution properties of $C(h, q)$. Yet we still know very little about this problem. In [3], the second author found that there exists some interesting connections between $C(h, q)$ and Kloosterman sums

$$
K(m, n ; q)=\sum_{b=1}^{q} e\left(\frac{m b+n \bar{b}}{q}\right)
$$

where $e(y)=e^{2 \pi i y}$. For example, if $q$ is a square-full number (i.e., $p \mid q$ if and only if $p^{2} \mid q$ ), then we have the following asymptotic formula

$$
\sum_{h=1}^{q} K(h, 1 ; q) C(h, q)=\frac{-1}{2 \pi^{2}} q \phi(q)+O\left(q \exp \left(\frac{3 \ln q}{\ln \ln q}\right)\right) .
$$

For general integer $q \geq 3$, the second author [4] proved the asymptotic formula

(2) $\sum_{h=1}^{q} K(h, 1 ; q) C(h, q)=\frac{-1}{2 \pi^{2}} q \phi(q) \prod_{p \| q}\left(1-\frac{1}{p(p-1)}\right)+O\left(q^{\frac{3}{2}+\epsilon}\right)$,

where $\epsilon$ be any fixed positive number. Moreover, the second author [5] studied the hybrid mean value of the Cochrane sums and the $r$-th Kloosterman sums

$$
K(m, n, r ; q)=\sum_{b=1}^{q} e\left(\frac{m b^{r}+n \bar{b}^{r}}{q}\right)
$$

and obtained the following asymptotic formula

$$
\sum_{h=1}^{q} K(h, 1, r ; p) C(h, q)=\frac{-1}{2 \pi^{2}} p^{2}+O\left(r p^{\frac{3}{2}} \ln ^{2} p\right)
$$


For any general positive integers $q \geq 3$ and $r$ with $l_{r}=(r, \phi(q))>1$, he [6] proved that

$$
\begin{aligned}
& \sum_{h=1}^{q} K(h, 1, r ; q) C(h, q) \\
= & \frac{-1}{2 \pi^{2}} q \phi(q) \prod_{p \|_{q}}\left(1-\frac{1}{p(p-1)}\right)+O\left(l_{r} q^{\frac{3}{2}+\epsilon}\right) .
\end{aligned}
$$

In [7], Professor Mordell introduced the hyper-Kloosterman sums as following:

$$
\begin{aligned}
& K l(h, k+1 ; q) \\
= & \sum_{a_{1}=1}^{q} \sum_{a_{2}=1}^{\prime} \cdots \sum_{a_{k}=1}^{q} e\left(\frac{a_{1}+a_{2}+\cdots+a_{k}+h \cdot \bar{a}_{1} \bar{a}_{2} \cdots \bar{a}_{k}}{q}\right) .
\end{aligned}
$$

About the hyper-Kloosterman sums, many scholars had studied about it before. Applications of the hyper-kloosterman sums were found in the estimation of Fourier coefficients of Maass forms [8] and the work on Selberg's e:genvalue conjecture [9]. On the other hand, Smith [10] had built some interesting connections between the hyper-Klooster-man sums and the Heibronn sums.

Following the spirit of [3], we found that there exists some interesting connections between the hyper-Kloosterman sums and the hyper Cochrane sums

$$
\begin{aligned}
& C(h, q ; m, k) \\
= & \sum_{a_{1}=1}^{q} \cdots \sum_{a_{k}=1}^{q} \bar{B}_{m_{1}}\left(\frac{\bar{a}_{1}}{q}\right) \bar{B}_{m_{k}}\left(\frac{\bar{a}_{k}}{q}\right) \bar{B}_{m_{k+1}}\left(\frac{h \cdot a_{1} \cdots a_{k}}{q}\right),
\end{aligned}
$$

and proved that the following mean value theorem

$$
\begin{aligned}
& \sum_{h=1}^{q} K l(h, k+1 ; q) C(h, q ; m, k) \\
= & \frac{(-1)^{k+1} 2^{k} m_{1} ! \cdots m_{k+1} ! \phi(q) q^{k}}{(2 \pi i)^{m_{1}+\cdots+m_{k+1}}} \prod_{p \| q}\left(1-\frac{p^{k}-1}{p^{k}(p-1)^{2}}\right) \\
& +O\left(q^{k+\epsilon}\right)
\end{aligned}
$$


holds for any odd numbers $m_{1}, m_{2}, \ldots, m_{k+1}$ (see [12]).

Similarly, we can define the $r$-th hyper-Kloosterman sums as following:

$$
\begin{aligned}
& K l(h, k+1, r ; q) \\
= & \sum_{a_{1}=1}^{q} \sum_{a_{2}=1}^{q} \cdots \sum_{a_{k}=1}^{q} e\left(\frac{a_{1}^{r}+a_{2}^{r}+\cdots+a_{k}^{r}+h \cdot \bar{a}_{1}^{r} \bar{a}_{2}^{r} \cdots \bar{a}_{k}^{r}}{q}\right) .
\end{aligned}
$$

It is obvious that $K l(h, k+1, r ; q)=K l(h, k+1 ; q)$ if $(r, \phi(q))=1$, so we suppose $(r, \phi(q))>1$. In this paper, we shall use the properties of Gauss sums, primitive characters and the mean value theorems of Dirichlet L-functions to study the hybrid mean value of the $r$-th hyper-Kloosterman sums $K l(h, k+1, r ; q)$ and the hyper Cochrane sums $C(h, q ; m, k)$, and give an interesting mean value formula. That is, we shall prove the following:

THEOREM. Let any positive integers $r$ and $q \geq 3$ with $l_{r}=(r, \phi(q))>$ 1. Then for any odd numbers $m_{1}, m_{2}, \ldots, m_{k+1}$, we have the asymptotic formula

$$
\begin{aligned}
& \sum_{h=1}^{q} K l(h, r, k+1 ; q) C(h, q ; m, k) \\
= & \frac{(-1)^{k+1} 2^{k} m_{1} ! \cdots m_{k+1} ! \phi(q) q^{k}}{(2 \pi i)^{m_{1}+\cdots+m_{k+1}}} \prod_{p \| q}\left(1-\frac{p^{k}-1}{p^{k}(p-1)^{2}}\right) \\
& +O\left(l_{r} q^{k+\frac{1}{2}+\epsilon}\right) .
\end{aligned}
$$

Taking $q=p$, an odd prime in our Theorem, we may immediately deduce the following

Corollary. For any odd prime $p$ and odd numbers $m_{1}, m_{2}, \ldots$, $m_{k+1}$, we have

$$
\begin{aligned}
& \sum_{h=1}^{p} K l(h, r, k+1 ; p) C(h, p ; m, k) \\
= & \frac{(-1)^{k+1} 2^{k} m_{1} ! \cdots m_{k+1} ! p^{k+1}}{(2 \pi i)^{m_{1}+\cdots+m_{k+1}}}+O\left(l_{r} p^{k+\frac{1}{2}+\epsilon}\right) .
\end{aligned}
$$




\section{Some lemmas}

To prove the theorem, we need the following several lemmas. For convenience, first we may define the $r$-th Gauss sums as

$$
G(n, \chi, r ; q)=\sum_{b=1}^{q} \chi(b) e\left(\frac{n b^{r}}{q}\right) .
$$

It is clear that $G(n, \chi, 1 ; q)=G(n, \chi)$ is the classical Gauss sums and we have the following

Lemma 1. For any positive integer $q$, let $\chi$ be a non-primitive character modulo $q$ and $\chi_{q} \Leftrightarrow \chi_{q^{*}}^{*}$. If $(n, q)>1$, we have

$$
G(n, \chi)= \begin{cases}\bar{\chi}^{*}\left(\frac{n}{(n, q)}\right) \chi^{*}\left(\frac{q}{q^{*}(n, q)}\right) \mu\left(\frac{q}{q^{*}(n, q)}\right) & \\ \times \phi(q) \phi^{-1}\left(\frac{q}{(n, q)}\right) \tau\left(\chi^{*}\right), & q^{*}=\frac{q_{1}}{\left(n, q_{1}\right)} \\ 0, & q^{*} \neq \frac{q_{1}}{\left(n, q_{1}\right)}\end{cases}
$$

where $q_{1}$ is the greatest divisor of $q$ that has the same prime factors as $q^{*}, \tau(\chi)=G(1, \chi)$, and $\mu(n)$ is the Möbius function.

If $(n, q)=1$, then we have

$$
G(n, \chi)=\bar{\chi}^{*}(n) \chi^{*}\left(\frac{q}{q^{*}}\right) \mu\left(\frac{q}{q^{*}}\right) \tau\left(\chi^{*}\right) .
$$

Proof. See [11].

LEMMA 2. Let $\chi$ be a character modulo $q$, generated by the primitive character $\chi_{m}$ modulo $m$. Then we have the identity

$$
\tau(\chi)=\chi_{m}\left(\frac{q}{m}\right) \mu\left(\frac{q}{m}\right) \tau\left(\chi_{m}\right) .
$$

Proof. See [11].

Lemma 3. Let any positive integers $h$ and $q \geq 3$ with $(h, q)=1$. Then for any odd numbers $m_{1}, m_{2}, \ldots, m_{k+1}$, we have

$$
\begin{aligned}
& C(h, q ; m, k) \\
= & \frac{(-2)^{k+1} m_{1} ! \cdots m_{k+1} !}{(2 \pi i)^{m_{1}+\cdots+m_{k+1} \phi(q)}} \\
& \times \sum_{\substack{\chi \bmod q \\
\chi(-1)=-1}} \bar{\chi}(h)\left\{\sum_{r_{1}=1}^{\infty} \frac{G\left(r_{1}, \chi\right)}{r_{1}^{m_{1}}}\right\} \cdots\left\{\sum_{r_{k+1}=1}^{\infty} \frac{G\left(r_{k+1}, \chi\right)}{r_{k+1}^{m_{k+1}}}\right\},
\end{aligned}
$$


where $\chi$ denotes a Dirichlet character modulo $q$ with $\chi(-1)=-1$.

Proof. From the orthogonality relation for character sums modulo $q$ we have

$$
\begin{aligned}
C(h, q ; m, k)= & \sum_{a_{1}=1}^{q} \bar{B}_{m_{1}}\left(\frac{\bar{a}_{1}}{q}\right) \cdots \sum_{a_{k}=1}^{q} \bar{B}_{m_{k}}\left(\frac{\bar{a}_{k}}{q}\right) \bar{B}_{m_{k+1}}\left(\frac{a_{1} \cdots a_{k} h}{q}\right) \\
= & \frac{1}{\phi(q)} \sum_{\chi \bmod q}\left\{\sum_{a_{1}=1}^{q} \chi\left(a_{1}\right) \bar{B}_{m_{1}}\left(\frac{a_{1}}{q}\right)\right\} \\
& \times \cdots \times\left\{\sum_{a_{k+1}=1}^{q} \chi\left(a_{k+1}\right) \bar{B}_{m_{k+1}}\left(\frac{a_{k+1} h}{q}\right)\right\} .
\end{aligned}
$$

Note that

$$
\bar{B}_{n}(x)=-\frac{n !}{(2 \pi i)^{n}} \sum_{\substack{r=-\infty \\ r \neq 0}}^{+\infty} \frac{e(x r)}{r^{n}},
$$

and for any integer $h$ with $(h, q)=1, G(h n, \chi)=\bar{\chi}(h) G(n, \chi)$. Then we have

$$
\begin{aligned}
& C(h, q ; m, k) \\
& =\frac{1}{\phi(q)} \sum_{\chi \bmod q}\left\{\sum_{a_{1}=1}^{q}\left(-\frac{m_{1} !}{(2 \pi i)^{m_{1}}}\right) \sum_{\substack{r_{1}=-\infty \\
r_{1} \neq 0}}^{+\infty} \chi\left(a_{1}\right) \frac{e\left(\frac{r_{1} a_{1}}{q}\right)}{r_{1}^{m_{1}}}\right\} \\
& \times \cdots \times\left\{\sum_{a_{k+1}=1}^{q}\left(-\frac{m_{k+1} !}{(2 \pi i)^{m_{k+1}}}\right) \sum_{\substack{r_{k+1}=-\infty \\
r_{k+1} \neq 0}}^{+\infty} \chi\left(a_{k+1}\right) \frac{e\left(\frac{r_{k+1} a_{k+1} h}{q}\right)}{r_{k+1}^{m_{k+1}}}\right\} \\
& =\frac{(-1)^{k+1} m_{1} ! \cdots \dot{m}_{k+1} !}{(2 \pi i)^{m_{1}+\cdots+m_{k+1} \phi(q)}} \sum_{\chi \bmod q}\left\{\sum_{\substack{r_{1}=-\infty \\
r_{1} \neq 0}}^{+\infty} \frac{1}{r_{1}^{m_{1}}} \sum_{a_{1}=1}^{q} \chi\left(a_{1}\right) e\left(\frac{r_{1} a_{1}}{q}\right)\right\} \\
& \times \cdots \times\left\{\sum_{\substack{r_{k+1}=-\infty \\
r_{k+1} \neq 0}}^{+\infty} \frac{1}{r_{k+1}^{m_{k+1}}} \sum_{a_{k+1}=1}^{q} \chi\left(a_{k+1}\right) e\left(\frac{r_{k+1} a_{k+1} h}{q}\right)\right\}
\end{aligned}
$$




$$
\begin{gathered}
=\frac{(-1)^{k+1} m_{1} ! \cdots m_{k+1} !}{(2 \pi i)^{m_{1}+\cdots+m_{k+1} \phi(q)}} \sum_{\chi}\left\{\sum_{\substack{\bmod q \\
r_{1}=-\infty \\
r_{1} \neq 0}}^{+\infty} \frac{G\left(r_{1}, \chi\right)}{r_{1}^{m_{1}}}\right\} \\
\times \cdots \times\left\{\sum_{\substack{r_{k+1}=-\infty \\
r_{k+1} \neq 0}}^{+\infty} \frac{G\left(h r_{k+1}, \chi\right)}{r_{k+1}^{m_{k+1}}}\right\} \\
=\frac{(-2)^{k+1} m_{1} ! \cdots m_{k+1} !}{(2 \pi i)^{m_{1}+\cdots+m_{k+1}} \phi(q)} \sum_{\substack{\chi \bmod \\
\chi(-1)=-1}} \bar{\chi}(h)\left\{\sum_{r_{1}=1}^{\infty} \frac{G\left(r_{1}, \chi\right)}{r_{1}^{m_{1}}}\right\} \\
\times \cdots \times\left\{\sum_{r_{k+1}=1}^{\infty} \frac{G\left(r_{k+1}, \chi\right)}{r_{k+1}^{m_{k+1}}}\right\} .
\end{gathered}
$$

This proves Lemma 3.

LEMMA 4. For any positive integers $q$ and $r>1$, let $l_{r}=(r, \phi(q))$ and $\chi_{1}$ be a $l_{\text {. }}$ th-order character modulo $q$. Then for any character $\chi$ modulo $q$, we have the identities

$$
\sum_{h=1}^{q} \bar{\chi}(h) K l(h, k+1,1 ; q)=\tau^{k+1}(\bar{\chi})
$$

and

$$
\sum_{h=1}^{q} \bar{\chi}(h) K l(h, k+1, r ; q)=\tau(\bar{\chi})\left(\tau(\bar{\chi})+\sum_{i=1}^{l_{r}-1} \tau\left(\bar{\chi} \chi_{1}^{i}\right)\right)^{k}
$$

Proof. From the properties of Gauss sums we have

$$
\begin{aligned}
& \sum_{h=1}^{q} \bar{\chi}(h) K l(h, k+1, r ; q) \\
= & \sum_{h=1}^{q} \bar{\chi}(h) \sum_{a_{1}=1}^{q} \sum_{a_{2}=1}^{q} \cdots \sum_{a_{k}=1}^{q} e\left(\frac{a_{1}^{r}+a_{2}^{r}+\cdots+a_{k}^{r}+h \cdot \bar{a}_{1}^{r} \bar{a}_{2}^{r} \cdots \bar{a}_{k}^{r}}{q}\right) \\
= & \sum_{h=1}^{q} \bar{\chi}(h) e\left(\frac{h}{q}\right) \sum_{a_{1}=1}^{q} \sum_{a_{2}=1}^{q}
\end{aligned}
$$




$$
\begin{aligned}
& \times \cdots \times \sum_{a_{k}=1}^{q} \bar{\chi}\left(a_{1}^{r} a_{2}^{r} \cdots a_{k}^{r}\right) e\left(\frac{a_{1}^{r}+a_{2}^{r}+\cdots+a_{k}^{r}}{q}\right) \\
= & \tau(\bar{\chi})\left(\sum_{b=1}^{k} \bar{\chi}\left(b^{r}\right) e\left(\frac{b^{r}}{q}\right)\right)^{k} \\
= & \tau(\bar{\chi}) G^{k}\left(1, \bar{\chi}^{r}, r ; q\right) .
\end{aligned}
$$

It is clear that $G(1, \bar{\chi}, 1 ; q)=\tau(\bar{\chi})$, so we have

$$
\sum_{h=1}^{q} \bar{\chi}(h) K l(h, k+1,1 ; q)=\tau^{k+1}(\bar{\chi})
$$

On the other hand, let $\chi_{1}$ be a $l_{r}$ th-order character modulo $q$. Then for any integer $1 \leq a \leq q$ with $(a, q)=1$, we have the identity

$$
1+\chi_{1}(a)+\cdots+\chi_{1}^{l_{r}-1}(a)= \begin{cases}l_{r}, & \text { if } a \text { is a } l_{r} \text { th residue } \bmod q \\ 0, & \text { otherwise }\end{cases}
$$

Therefore

$$
\begin{aligned}
G\left(1, \bar{\chi}^{r}, r ; q\right) & =\sum_{b=1}^{k} \bar{\chi}\left(b^{r}\right) e\left(\frac{b^{r}}{q}\right) \\
& =\sum_{b=1}^{q}\left(1+\chi_{1}(b)+\cdots+\chi_{1}^{l_{r}-1}(b)\right) \bar{\chi}(b) e\left(\frac{b}{q}\right) \\
& =\tau(\bar{\chi})+\sum_{i=1}^{l_{r}-1} \tau\left(\bar{\chi} \chi_{1}^{i}\right) .
\end{aligned}
$$

This proves Lemma 4.

LEMMA 5. Let $q$ and $r$ be integers with $q \geq 2$ and $(r, q)=1, \chi$ be a Dirichlet character modulo $q$. Then we have the identities

$$
\sum_{\chi \bmod q}^{*} \chi(r)=\sum_{d \mid(q, r-1)} \mu\left(\frac{q}{d}\right) \phi(d)
$$

and

$$
J(q)=\sum_{d \mid q} \mu(d) \phi\left(\frac{q}{d}\right)
$$

where $\sum_{\chi \bmod q}^{*}$ denotes the summation over all primitive characters modulo $q$ and $J(q)$ denotes the number of primitive characters modulo $q$. 
Proof. This is Lemma 3 of [3].

LEMMA 6. For any positive integers $r$ and $q \geq 3$ with $l_{r}=(r, \phi(q))>$ 1 , let $q=u v$, where $(u, v)=1, u$ be a square-full number or $u=1, v$ be a square-free number, $\chi_{1}$ be a $l_{r}$ th-order character modulo $q$. For any positive integers $t_{1}, t_{2}, \ldots, t_{k+1}$, if we let

$$
\begin{aligned}
\Phi= & \sum_{d \mid v} \sum_{d_{1} \mid \frac{v}{d}} \cdots \sum_{d_{k+1} \mid \frac{v}{d}} \frac{u^{k} d^{k} \mu\left(d_{1}\right) \cdots \mu\left(d_{k}\right) \mu\left(\frac{v}{d d_{k+1}}\right)}{d_{1}^{t_{1}} \cdots d_{k+1}^{t_{k+1}} \phi\left(\frac{q}{d_{1}}\right) \cdots \phi\left(\frac{q}{d_{k+1}}\right)} \\
& \times \sum_{\chi \bmod u d}^{*} \bar{\chi}\left(d_{1} \cdots d_{k}\right) \chi\left(\frac{v}{d d_{k+1}}\right) \\
& \times L\left(t_{1}, \bar{\chi}\right) \cdots L\left(t_{k+1}, \bar{\chi}\right) \sum_{a=1}^{u d} \chi(a) e\left(\frac{a}{u d}\right) \\
& \times \sum_{i=1}^{l_{r}-1} \sum_{l=0}^{q / u d-1} \sum_{m=1}^{u d} \chi_{1}^{i}(l u d+m) \bar{\chi}(m) e\left(\frac{l u d+m}{q}\right)
\end{aligned}
$$

then we have the following estimate

$$
\Phi \ll l_{r} q^{\frac{1}{2}+\epsilon}
$$

Proof. Without loss of generality we can assume $t=\min \left(t_{1}, t_{2}, \ldots\right.$, $\left.t_{k+1}\right)$. We now let

$$
r_{k+1}(n)=\sum_{d_{1} d_{2} \cdots d_{k+1}=n} d_{1}^{t-t_{1}} d_{2}^{t-t_{2}} \cdots d_{k+1}^{t-t_{k+1}}
$$

Then for any parameter $N \geq u d$ and non-principal character $\chi$ modulo $u d$, applying Abel's identity we have

$$
\begin{aligned}
L\left(t_{1}, \bar{\chi}\right) \cdots L\left(t_{k+1}, \bar{\chi}\right) & =\sum_{n=1}^{\infty} \frac{\bar{\chi}(n) r_{k+1}(n)}{n^{t}} \\
& =\sum_{1 \leq n \leq N} \frac{\bar{\chi}(n) r_{k+1}(n)}{n^{t}}+t \int_{N}^{\infty} \frac{A(y, \bar{\chi})}{y^{t+1}} d y
\end{aligned}
$$


where $A(y, \bar{\chi})=\sum_{N<n \leq y} \bar{\chi}(n) r_{k+1}(n)$.

Note that by using induction, we can prove the following estimate

$$
\sum_{\substack{\chi \bmod u d \\ \chi(-1)=-1}}|A(y, \bar{\chi})| \ll y^{1-\left(2 / 2^{k+1}\right)+\epsilon} \phi^{3 / 2}(u d)
$$

(see [12]).

Hence we have

$$
\begin{aligned}
& \sum_{d \mid v} \sum_{d_{1} \mid \frac{v}{d}} \cdots \sum_{d_{k+1} \mid \frac{v}{d}} \frac{u^{k} d^{k} \mu\left(d_{1}\right) \cdots \mu\left(d_{k}\right) \mu\left(\frac{v}{d d_{k+1}}\right)}{d_{1}^{t_{1}} \cdots d_{k+1}^{t_{k+1}} \phi\left(\frac{q}{d_{1}}\right) \cdots \phi\left(\frac{q}{d_{k+1}}\right)} \\
& \times \sum_{i=1}^{l_{r}-1} \sum_{l=0}^{q / u d-1} \sum_{m=1}^{u d} \chi_{1}^{i}(l u d+m) \\
& \times e\left(\frac{l u d+m}{q}\right) \sum_{a=1}^{u d} e\left(\frac{a m}{u d}\right) \\
& \times \sum_{\substack{\chi \bmod u d \\
\chi(-1)=-1}}^{*} \bar{\chi}\left(d_{1} \cdots d_{k} m\right) \chi\left(d_{k+1} a m\right) t \int_{N}^{\infty} \frac{A(y, \bar{\chi})}{y^{t+1}} d y \\
& \ll l_{r} q^{1+\epsilon} \int_{N}^{\infty} \frac{t}{y^{t+1}}\left(\sum_{\substack{\chi \bmod q \\
\chi(-1)=-1}}|A(y, \bar{\chi})|\right) d y \\
& \ll l_{r} q^{1+\epsilon} \int_{N}^{\infty} \frac{y^{1-\frac{1}{2^{k}+\epsilon}} \phi^{3 / 2}(q)}{y^{t+1}} d y \\
& \ll \frac{l_{r} q^{\frac{5}{2}+\epsilon}}{(\sqrt{N})^{2 t+2^{1-k}-2}} .
\end{aligned}
$$

Combining the above we have

$$
\begin{aligned}
\Phi= & \sum_{d \mid v} \sum_{d_{1} \mid \frac{v}{d}} \cdots \sum_{d_{k+1} \mid \frac{v}{d}} \frac{u^{k} d^{k} \mu\left(d_{1}\right) \cdots \mu\left(d_{k}\right) \mu\left(\frac{v}{d d_{k+1}}\right)}{d_{1}^{t_{1}} \cdots d_{k+1}^{t_{k+1}} \phi\left(\frac{q}{d_{1}}\right) \cdots \phi\left(\frac{q}{d_{k+1}}\right)} \\
& \times \sum_{i=1}^{l_{r}-1} \sum_{l=0}^{q / u d-1} \sum_{m=1}^{u d} \chi_{1}^{i}(l u d+m) e\left(\frac{l u d+m}{q}\right)
\end{aligned}
$$


(6)

$$
\begin{aligned}
& \times \sum_{a=1}^{u d} e\left(\frac{a m}{u d}\right) \sum_{1 \leq n \leq N} \frac{r_{k+1}(n)}{n^{t}} \\
& \times \sum_{\substack{\chi \bmod u d \\
\chi(-1)=-1}}^{*} \bar{\chi}\left(d_{1} \cdots d_{k} n m\right) \chi\left(d_{k+1} a m\right) \\
& +O\left(\frac{l_{r} q^{\frac{5}{2}+\epsilon}}{(\sqrt{N})^{2 t+2^{1-k}-2}}\right) \text {. }
\end{aligned}
$$

Note that for any integer $a$ with $(a, q)=1$, from Lemma 5 we have

$$
\begin{aligned}
\sum_{\substack{\chi \bmod q \\
\chi(-1)=-1}}^{*} \chi(a) & =\frac{1}{2} \sum_{\chi \bmod q}^{*}(1-\chi(-1)) \chi(a) \\
& =\frac{1}{2} \sum_{\chi \bmod q}^{*} \chi(a)-\frac{1}{2} \sum_{\chi \bmod q}^{*} \chi(-a) \\
& =\frac{1}{2} \sum_{u \mid(q, a-1)} \mu\left(\frac{q}{u}\right) \phi(u)-\frac{1}{2} \sum_{u \mid(q, a+1)} \mu\left(\frac{q}{u}\right) \phi(u) .
\end{aligned}
$$

Therefore by formula (6) and $\sum_{d \mid n} f(d)=\sum_{d \mid n} f\left(\frac{n}{d}\right)$ we have

$$
\begin{aligned}
\Phi=\sum_{d \mid v} & \sum_{d_{1} \mid \frac{v}{d}} \\
& \times \cdots \times \sum_{d_{k+1} \mid \frac{v}{d}} \frac{u^{k} d^{k} \mu\left(d_{1}\right) \cdots \mu\left(d_{k+1}\right)}{d_{1}^{t_{1}} \cdots d_{k}^{t_{k}}\left(\frac{v}{d d_{k+1}}\right)^{t_{k+1}} \phi\left(\frac{q}{d_{1}}\right) \cdots \phi\left(\frac{q}{d_{k}}\right) \phi\left(\frac{q d d_{k+1}}{v}\right)} \\
& \times \sum_{i=1}^{l_{r}-1} \sum_{l=0}^{q / u d-1} \sum_{m=1}^{u d} \chi_{1}^{i}(l u d+m) e\left(\frac{l u d+m}{q}\right) \sum_{a=1}^{u d} e\left(\frac{a m}{u d}\right) \\
& \times \sum_{1 \leq n \leq N} \frac{r_{k+1}(n)}{n^{t}} \sum_{\substack{\bmod u d \\
\chi(-1)=-1}}^{*} \bar{\chi}\left(d_{1} \cdots d_{k} n m\right) \chi\left(d_{k+1} a m\right) \\
& +O\left(\frac{l_{r} q^{\frac{5}{2}+\epsilon}}{(\sqrt{N})^{2 t+2^{1-k}-2}}\right)
\end{aligned}
$$




$$
\begin{aligned}
& =\frac{1}{2} \sum_{d \mid v} \sum_{d_{1} \mid \frac{v}{d}} \\
& \times \cdots \times \cdots \sum_{d_{k+1} \mid \frac{v}{d}} \frac{u^{k} d^{k} \mu\left(d_{1}\right) \cdots \mu\left(d_{k+1}\right)}{d_{1}^{t_{1}} \cdots d_{k}^{t_{k}}\left(\frac{v}{d d_{k+1}}\right)^{t_{k+1}} \phi\left(\frac{q}{d_{1}}\right) \cdots \phi\left(\frac{q}{d_{k}}\right) \phi\left(\frac{q d d_{k+1}}{v}\right)} \\
& \times \sum_{i=1}^{l_{r}-1} \sum_{l=0}^{q / u d-1} \sum_{m=1}^{u d} \chi_{1}^{i}(l u d+m) e\left(\frac{l u d+m}{q}\right) \sum_{a=1}^{u d} e\left(\frac{a m}{u d}\right)
\end{aligned}
$$

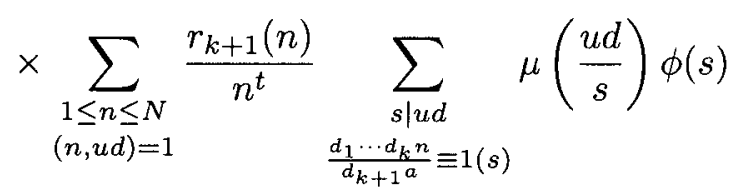

$$
\begin{aligned}
& -\frac{1}{2} \sum_{d \mid v} \sum_{d_{1} \mid \frac{v}{d}} \\
& \times \cdots \times \sum_{d_{k+1} \mid \frac{v}{d}} \frac{u^{k} d^{k} \mu\left(d_{1}\right) \cdots \mu\left(d_{k+1}\right)}{d_{1}^{t_{1}} \cdots d_{k}^{t_{k}}\left(\frac{v}{d d_{k+1}}\right)^{t_{k+1}} \phi\left(\frac{q}{d_{1}}\right) \cdots \phi\left(\frac{q}{d_{k}}\right) \phi\left(\frac{q d d_{k+1}}{v}\right)} \\
& \times \sum_{i=1}^{l_{r}-1} \sum_{l=0}^{q / u d-1} \sum_{m=1}^{u d} \chi_{1}^{i}(l u d+m) e\left(\frac{l u d+m}{q}\right) \sum_{a=1}^{u d} e\left(\frac{a m}{u d}\right) \\
& \times \sum_{\substack{1 \leq n \leq N \\
(n, u d)=1}} \frac{r_{k+1}(n)}{n^{t}} \sum_{\substack{s \mid u d \\
\frac{d_{1} \cdots d_{k} n}{d_{k+1} a} \equiv-1(s)}} \mu\left(\frac{u d}{s}\right) \phi(s) \\
& +O\left(\frac{l_{r} q^{\frac{5}{2}+\epsilon}}{(\sqrt{N})^{2 t+2^{1-k}-2}}\right) \\
& =\frac{1}{2} \sum_{d \mid v} \sum_{d_{1} \mid \frac{v}{d}} \\
& \times \cdots \times \sum_{d_{k+1} \mid \frac{v}{d}} \frac{u^{k} d^{k} \mu\left(d_{1}\right) \cdots \mu\left(d_{k+1}\right)}{d_{1}^{t_{1}} \cdots d_{k}^{t_{k}}\left(\frac{v}{d d_{k+1}}\right)^{t_{k+1}} \phi\left(\frac{q}{d_{1}}\right) \cdots \phi\left(\frac{q}{d_{k}}\right) \phi\left(\frac{q d d_{k+1}}{v}\right)} \\
& \times \sum_{i=1}^{l_{r}-1} \sum_{l=0}^{q / u d-1} \sum_{m=1}^{u d} \chi_{1}^{i}(l u d+m) e\left(\frac{l u d+m}{q}\right) \sum_{\substack{a=1 \\
d_{1} \cdots d_{k} \mid d_{k+1} a}}^{u d} e\left(\frac{a m}{u d}\right) \\
& \times \sum_{s \mid u d} \mu\left(\frac{u d}{s}\right) \phi(s) \sum_{l=0}^{\frac{d_{1} \cdots d_{k} N}{d_{k+1} a s}} \frac{r_{k+1}\left(\frac{(l s+1) a d_{k+1}}{d_{1} \cdots d_{k}}\right)}{\left(\frac{(l s+1) a d_{k+1}}{d_{1} \cdots d_{k}}\right)^{t}}-\frac{1}{2} \sum_{d \mid v} \sum_{d_{1} \mid \frac{v}{d}}
\end{aligned}
$$




$$
\begin{aligned}
& \times \cdots \times \sum_{d_{k+1} \mid \frac{v}{d}} \frac{u^{k} d^{k} \mu\left(d_{1}\right) \cdots \mu\left(d_{k+1}\right)}{d_{1}^{t_{1}} \cdots d_{k}^{t_{k}}\left(\frac{v}{d d_{k+1}}\right)^{t_{k+1}} \phi\left(\frac{q}{d_{1}}\right) \cdots \phi\left(\frac{q}{d_{k}}\right) \phi\left(\frac{q d d_{k+1}}{v}\right)} \\
& \times \sum_{i=1}^{l_{r}-1} \sum_{l=0}^{q / u d-1} \sum_{m=1}^{u d} \chi_{1}^{i}(l u d+m) e\left(\frac{l u d+m}{q}\right) \sum_{\substack{a=1 \\
d_{1} \cdots d_{k} \mid d_{k+1} a}}^{\prime d} e\left(\frac{a m}{u d}\right) \\
& \times \sum_{s \mid u d} \mu\left(\frac{u d}{s}\right) \phi(s) \sum_{l=0}^{\frac{d_{1} \cdots d_{k} N}{d_{k+1}{ }^{a s}}} \frac{r_{k+1}\left(\frac{(l s-1) a d_{k+1}}{d_{1} \cdots d_{k}}\right)}{\left(\frac{(l s-1) a d_{k+1}}{d_{1} \cdots d_{k}}\right)^{t}} \\
& +O\left(\frac{l_{r} q^{\frac{5}{2}+\epsilon}}{(\sqrt{N})^{2 t+2^{1-k}-2}}\right) .
\end{aligned}
$$

From the definition of $t$, we know that all the terms in the above have the highest order only in the case that $t=t_{1}=\cdots=t_{k+1}$, so we have

$$
\begin{aligned}
& \Phi \ll \sum_{d \mid v} \sum_{d_{1} \mid \frac{v}{d}} \cdots \sum_{d_{k+1} \mid \frac{v}{d}} \frac{u^{k} d^{k} \mu\left(d_{1}\right) \cdots \mu\left(d_{k+1}\right)}{\left(\frac{v}{d}\right)^{t_{k+1}} \phi\left(\frac{q}{d_{1}}\right) \cdots \phi\left(\frac{q}{d_{k}}\right) \phi\left(\frac{q d d_{k+1}}{v}\right)} \\
& \times \sum_{i=1}^{l_{r}--q / u d-1} \sum_{l=0}^{u d} \sum_{m=1}^{\prime} \chi_{1}^{i}(l u d+m) e\left(\frac{l u d+m}{q}\right) \\
& \times \sum_{\substack{a=1 \\
d_{1} \cdots d_{k} \mid d_{k+1} a}}^{u d} e\left(\frac{l u d+m}{q}\right) \frac{r_{k+1}\left(\frac{d_{k+1} a}{d_{1} \cdots d_{k}}\right)}{a^{t_{k+1}}} \sum_{s \mid u d} \mu\left(\frac{u d}{s}\right) \phi(s) \\
& +O\left(\sum_{d \mid v} \sum_{d_{1} \mid \frac{v}{d}} \cdots \sum_{d_{k+1} \mid \frac{v}{d}} \frac{u^{k} d^{k}}{\phi^{k}(q) \phi(u d)} \cdot l_{r} \cdot q\right. \\
& \left.\times \sum_{a=1}^{u d} \frac{1}{a^{t_{k+1}}} \cdot \sum_{s \mid u d} \phi(s) \sum_{l=1}^{\frac{d_{1} \cdots d_{k} N}{d_{k+1} a s}} \frac{N^{\epsilon_{1}}}{(l s+1)^{t_{k+1}}}\right) \\
& +O\left(\sum_{d \mid v} \sum_{d_{1} \mid \frac{v}{d}} \cdots \sum_{d_{k+1} \mid \frac{v}{d}} \frac{u^{k} d^{k}}{\phi^{k}(q) \phi(u d)} \cdot l_{r} \cdot q\right. \\
& \left.\times \sum_{a=1}^{u d} \frac{1}{a^{t_{k+1}}} \cdot \sum_{s \mid u d} \phi(s) \sum_{l=1}^{\frac{d_{1} \cdots d_{k} N}{d_{k+1} a^{s}}} \frac{N^{\epsilon_{1}}}{(l s-1)^{t_{k+1}}}\right)
\end{aligned}
$$




$$
\begin{aligned}
& +O\left(\frac{l_{r} q^{\frac{5}{2}+\epsilon}}{(\sqrt{N})^{2 t+2^{1-k}-2}}\right) \\
& \ll \sum_{d \mid v} \sum_{d_{1} \mid \frac{v}{d}} \cdots \sum_{d_{k+1} \mid \frac{v}{d}} \frac{u^{k} d^{k} \mu\left(d_{1}\right) \cdots \mu\left(d_{k+1}\right)}{\left(\frac{v}{d}\right)^{t_{k+1}} \phi\left(\frac{q}{d_{1}}\right) \cdots \phi\left(\frac{q}{d_{k}}\right) \phi\left(\frac{q d d_{k+1}}{v}\right)} \\
& \times \sum_{i=1}^{l_{r}-1} \sum_{l=0}^{q / u d-1} \sum_{m=1}^{u d} \chi_{1}^{i}(l u d+m) \\
& \times e\left(\frac{l u d+m}{q}\right) \sum_{a=1}^{\prime}{ }^{\prime} e\left(\frac{\frac{q}{u d} \cdot a(l u d+m)}{q}\right) \\
& \times \frac{r_{k+1}\left(d_{k+1} a / d_{1} \cdots d_{k}\right)}{a^{t_{k+1}}} \sum_{s \mid u d} \mu\left(\frac{u d}{s}\right) \phi(s) \\
& +O\left(\frac{l_{r} q^{\frac{5}{2}+\epsilon}}{(\sqrt{N})^{2 t+2^{1-k}-2}}\right)+O\left(l_{r} N^{\epsilon_{1}}\right)
\end{aligned}
$$

where we have used the estimate $r_{k+1}(n) \ll n^{\epsilon_{1}}$.

Now taking $N=q^{5 \times 2^{k-1}}$ in the above, and note the identity $J(u)=$ $\phi^{2}(u) / u$ if $u$ is a square-full number, we can immediately obtain the following

$$
\begin{aligned}
\Phi \ll & \sum_{d \mid v} \sum_{d_{1} \mid \frac{v}{d}} \cdots \sum_{d_{k+1} \mid \frac{v}{d}} \frac{u^{k} d^{k} \mu\left(d_{1}\right) \cdots \mu\left(d_{k+1}\right)}{\left(\frac{v}{d}\right)^{t_{k+1}} \phi\left(\frac{q}{d_{1}}\right) \cdots \phi\left(\frac{q}{d_{k}}\right) \phi\left(\frac{q d d_{k+1}}{v}\right)} \\
& \times \sum_{i=1}^{l_{r}-1} \sum_{b=1}^{q} \chi_{1}^{i}(b) e\left(\frac{b}{q}\right) \sum_{\substack{a=1 \\
d_{1} \cdots d_{k} \mid d_{k+1} a}}^{\prime \prime} e\left(\frac{\frac{a v}{d} b}{q}\right) \frac{r_{k+1}\left(d_{k+1} a / d_{1} \cdots d_{k}\right)}{a^{t_{k+1}}} \\
& \times \sum_{s \mid u d} \mu\left(\frac{u d}{s}\right) \phi(s)+O\left(l_{r} q^{\epsilon}\right) \\
\ll & \sum_{d \mid v} \sum_{d_{1} \mid \frac{v}{d}} \cdots \sum_{d_{k+1} \mid \frac{v}{d}} \frac{u^{k} d^{k} J(u d) \mu\left(d_{1}\right) \cdots \mu\left(d_{k+1}\right)}{\left(\frac{v}{d}\right)^{t_{k+1}} \phi\left(\frac{q}{d_{1}}\right) \cdots \phi\left(\frac{q}{d_{k}}\right) \phi\left(\frac{q d d_{k+1}}{v}\right)} \\
& \times \sum_{\substack{a=1 \\
d_{1} \cdots d_{k} \mid d_{k+1} a}}^{r_{k+1}\left(d_{k+1} a / d_{1} \cdots d_{k}\right)} \\
a^{t_{k+1}} & \sum_{i=1}^{l_{r}-1} G\left(1+a v / d, \chi_{1}^{i}\right)+O\left(l_{r} q^{\epsilon}\right)
\end{aligned}
$$




$$
=: \Psi+O\left(l_{r} q^{\epsilon}\right) \text {. }
$$

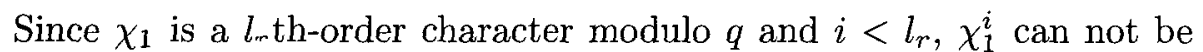
principal character modulo $q$. From the properties of Gauss sums we get

$$
\left|G\left(1+a v / d, \chi_{1}^{i}\right)\right| \ll(1+a v / d, q) q^{\frac{1}{2}+\epsilon} .
$$

So we have

$$
\begin{aligned}
\Psi & \ll \sum_{d \mid v} \sum_{d_{1} \mid \frac{v}{d}} \cdots \sum_{d_{k+1} \mid \frac{v}{d}} \frac{(u d)^{k+1}}{\phi^{k+1}(u d)} \cdot l_{r} q^{\frac{1}{2}+\epsilon} \cdot \sum_{a=1}^{u d} \frac{(1+a v / d, q)}{(a v / d)^{t_{k+1}}} \\
& \ll \sum_{d \mid v} l_{r} q^{\frac{1}{2}+\epsilon} \sum_{s \mid q} \sum_{\substack{a=1 \\
s \mid 1+a v / d}} \frac{s}{(a v / d)^{t_{k+1}}} \\
& \ll l_{r} q^{\frac{1}{2}+\epsilon} \sum_{s \mid q} \sum_{1 \leq l \leq \frac{q+1}{s}} \frac{s}{(l s-1)^{t_{k+1}}} \ll l_{r} q^{\frac{1}{2}+\epsilon} .
\end{aligned}
$$

This completes the proof of Lemma 6 .

\section{Proof of the theorem}

In this section, we shall complete the proof of the theorem. For any positive integers $r$ and $q \geq 3$ with $l_{r}=(r, \phi(q))>1$, let $\chi_{1}$ be a $l_{r}$ thorder character modulo $q$. Then from Lemma 4 we have

(7)

$$
\begin{aligned}
& \sum_{h=1}^{q} \bar{\chi}(h) K l(h, k+1, r ; q) \\
= & \sum_{a_{1}=1}^{q} \sum_{a_{2}=1}^{q} \cdots \sum_{a_{k}=1}^{q} \sum_{h=1}^{q} \bar{\chi}(h) e\left(\frac{a_{1}^{r}+a_{2}^{r}+\cdots+a_{k}^{r}+h \cdot \bar{a}_{1}^{r} \bar{a}_{2}^{r} \cdots \bar{a}_{k}^{r}}{q}\right) \\
= & \tau(\bar{\chi})\left(\tau(\bar{\chi})+\sum_{i=1}^{l_{r}-1} \tau\left(\bar{\chi} \chi_{1}^{i}\right)\right)^{k} .
\end{aligned}
$$


Hence from Lemma 3 and 4 we have

$$
\begin{aligned}
& \sum_{h=1}^{q} K l(h, k+1, r ; q) C(h, q ; m, k) \\
& =\frac{(-2)^{k+1} m_{1} ! \cdots m_{k+1} !}{(2 \pi i)^{m_{1}+\cdots+m_{k+1}} \phi(q)} \sum_{\substack{\chi \bmod \\
\chi(-1)=-1}}\left(\sum_{h=1}^{q} \bar{\chi}(h) K l(h, k+1, r ; q)\right) \\
& \times\left\{\sum_{r_{1}=1}^{\infty} \frac{G\left(r_{1}, \chi\right)}{r_{1}^{m_{1}}}\right\} \times \cdots \times\left\{\sum_{r_{k+1}=1}^{\infty} \frac{G\left(r_{k+1}, \chi\right)}{r_{k+1}^{m_{k+1}}}\right\} \\
& =\sum_{h=1}^{q} K l(h, k+1 ; q) C(h, q ; m, k)+\frac{(-2)^{k+1} m_{1} ! \cdots m_{k+1} !}{(2 \pi i)^{m_{1}+\cdots+m_{k+1} \phi(q)}} \\
& \times \sum_{\substack{\chi \bmod q \\
\chi(-1)=-1}} C_{k}^{1} \cdot \tau^{k}(\bar{\chi}) \sum_{i=1}^{l_{r}-1} \tau\left(\bar{\chi} \chi_{1}^{i}\right) \\
& \times\left\{\sum_{r_{1}=1}^{\infty} \frac{G\left(r_{1}, \chi\right)}{r_{1}^{m_{1}}}\right\} \times \cdots \times\left\{\sum_{r_{k+1}=1}^{\infty} \frac{G\left(r_{k+1}, \chi\right)}{r_{k+1}^{m_{k+1}}}\right\} \\
& +\frac{(-2)^{k+1} m_{1} ! \cdots m_{k+1} !}{(2 \pi i)^{m_{1}+\cdots+m_{k+1} \phi(q)}} \\
& \times \sum_{j=2}^{k} \sum_{\substack{\chi \bmod q \\
\chi(-1)=-1}} C_{k}^{j} \cdot \tau^{k-j+1}(\bar{\chi})\left(\sum_{i=1}^{l_{r}-1} \tau\left(\bar{\chi} \chi_{1}^{i}\right)\right)^{j} \\
& \times\left\{\sum_{r_{1}=1}^{\infty} \frac{G\left(r_{1}, \chi\right)}{r_{1}^{m_{1}}}\right\} \times \cdots \times\left\{\sum_{r_{k+1}=1}^{\infty} \frac{G\left(r_{k+1}, \chi\right)}{r_{k+1}^{m_{k+1}}}\right\} \\
& \equiv \sum_{h=1}^{q} K l(h, k+1 ; q) C(h, q ; m, k)+E_{1}+E_{2} \text {, }
\end{aligned}
$$

where $C_{k}^{j}=\frac{k !}{(k-j) ! j !}$.

We now let $q=u v$, where $(u, v)=1, u$ be a square-full number or $u=1, v$ be a square-free number. Note that $\chi^{*}\left(\frac{q}{m}\right) \mu\left(\frac{q}{m}\right) \neq 0$ if and 
only if $m=u d$, where $d \mid v$. Then from Lemma 1 and Lemma 2 we have

$$
\begin{aligned}
& E_{1}=\frac{k \cdot(-2)^{k+1} m_{1} ! \cdots m_{k+1} !}{(2 \pi i)^{m_{1}+\cdots+m_{k+1} \phi(q)}} \sum_{\substack{\chi \bmod q \\
\chi(-1)=-1}} \tau^{k}(\bar{\chi}) \sum_{i=1}^{l_{r}-1} \tau\left(\bar{\chi} \chi_{1}^{i}\right) \\
& \times\left\{\sum_{r_{1}=1}^{\infty} \frac{G\left(r_{1}, \chi\right)}{r_{1}^{m_{1}}}\right\} \times \cdots \times\left\{\sum_{r_{k+1}=1}^{\infty} \frac{G\left(r_{k+1}, \chi\right)}{r_{k+1}^{m_{k+1}}}\right\} \\
& =\frac{k \cdot(-2)^{k+1} m_{1} ! \cdots m_{k+1} !}{(2 \pi i)^{m_{1}+\cdots+m_{k+1} \phi(q)}} \sum_{d \mid v} \sum_{\chi \bmod u d}^{*} \\
& \chi(-1)=-1 \\
& \left\{\sum_{d_{1} \mid \frac{v}{d}} \frac{\phi(q) \chi\left(\frac{v}{d d_{1}}\right) \mu\left(\frac{v}{d d_{1}}\right) \tau(\chi) L\left(m_{1}, \bar{\chi}\right)}{d_{1}^{m_{1}} \phi\left(\frac{q}{d_{1}}\right)}\right\} \\
& \times \cdots \times\left\{\sum_{d_{k+1} \mid \frac{v}{d}} \frac{\phi(q) \chi\left(\frac{v}{d d_{k+1}}\right) \mu\left(\frac{v}{d d_{k+1}}\right) \tau(\chi) L\left(m_{k+1}, \bar{\chi}\right)}{d_{k+1}^{m_{k+1}} \phi\left(\frac{q}{d_{k+1}}\right)}\right\} \\
& \times \bar{\chi}^{k}\left(\frac{v}{d}\right) \mu^{k}\left(\frac{v}{d}\right) \tau^{k}(\bar{\chi}) \\
& =\frac{k \cdot(-2)^{k+1} m_{1} ! \cdots m_{k+1} ! \phi^{k}(q)}{(2 \pi i)^{m_{1}+\cdots+m_{k+1}}} \\
& \times \sum_{d \mid v} \sum_{d_{1} \mid \frac{v}{d}} \cdots \sum_{d_{k+1} \mid \frac{v}{d}} \frac{u^{k} d^{k} \mu\left(d_{1}\right) \cdots \mu\left(d_{k}\right) \mu\left(\frac{v}{d d_{k+1}}\right)}{d_{1}^{t_{1}} \cdots d_{k+1}^{t_{k+1}} \phi\left(\frac{q}{d_{1}}\right) \cdots \phi\left(\frac{q}{d_{k+1}}\right)} \\
& \times \sum_{\substack{\chi \bmod u d \\
\chi(-1)=-1}}^{*} \bar{\chi}\left(d_{1} \cdots d_{k}\right) \chi\left(\frac{v}{d d_{k+1}}\right) L\left(t_{1}, \bar{\chi}\right) \cdots L\left(t_{k+1}, \bar{\chi}\right) \\
& \times \sum_{a=1}^{u d} \chi(a) e\left(\frac{a}{u d}\right) \sum_{i=1}^{l_{r}-1} \sum_{l=0}^{q / u d-1} \sum_{m=1}^{u d} \chi_{1}^{i}(l u d+m) \bar{\chi}(m) e\left(\frac{l u d+m}{q}\right)
\end{aligned}
$$

where we have used the identities that

$$
\bar{\chi}\left(\frac{v}{d}\right)=\bar{\chi}\left(\frac{v}{d d_{1}}\right) \bar{\chi}\left(d_{1}\right), \quad \mu\left(\frac{v}{d}\right)=\mu\left(\frac{v}{d d_{1}}\right) \mu\left(d_{1}\right)
$$

and

$$
\tau\left(\chi^{*}\right) \tau\left(\bar{\chi}^{*}\right)=-m
$$


where $\chi^{*}$ is a primitive character modulo $m$ and $\chi(-1)=-1$. So from Lemma 6 we have the estimate

$$
E_{1} \ll l_{r} q^{k+\frac{1}{2}+\epsilon}
$$

Using the similar method of proving Lemma 6 , we may obtain the following estimate

$$
E_{2} \ll l_{r} q^{k+\frac{1}{2}+\epsilon}
$$

Therefore from (5), (8), (9) and (10), we may immediately obtain

$$
\begin{aligned}
& \sum_{h=1}^{q} K l(h, k+1, r ; q) C(h, q ; m, k) \\
= & \sum_{h=1}^{q} K l(h, k+1 ; q) C(h, q ; m, k)+O\left(l_{r} q^{k+\frac{1}{2}+\epsilon}\right) \\
= & \frac{(-1)^{k+1} 2^{k} m_{1} ! \cdots m_{k+1} ! \phi(q) q^{k}}{(2 \pi i)^{m_{1}+\cdots+m_{k+1}}} \prod_{p \| q}\left(1-\frac{p^{k}-1}{p^{k}(p-1)^{2}}\right) \\
& +O\left(l_{r} q^{k+\frac{1}{2}+\epsilon}\right) .
\end{aligned}
$$

This completes the proof of the theorem.

ACKNOWLEDGMENTS. The authors express their gratitude to the anonymous referee for his very helpful and detailed comments on the original manuscripts.

\section{References}

[1] D. Bump, W. Duke, J. Hoffstein, and H. Iwaniec, An estimate for the Hecke eigenvalues of Maass forms, Internat.Math. Res. Notices (1992), no. 4, 75-81.

[2] W. Luo, Z. Rudnick, and P. Sarnak, On Selberg's eigenvalue conjecture, Geom. Funct. Anal. 5 (1995), no. 2, 387-401.

[3] L. J. Mordell, On a special polynomial congruence and exponential sums : in "Calcutta Math. Soc. Golden Jubilee Commemoration Volume" Part 1, Calcutta Math. Soc., Calcutta, 1963, pp. 29-32.

[4] Chengdong Pan and Chengbiao Pan, Goldbach's Conjecture, Science Press, Beijing, 1981.

[5] R. A. Smith, On n-dimensional Kloosterman sums, J. Number Theory 11 (1979), no. 3, 324-343. 
[6] M. Xie and W. Zhang, On the 2k-th mean value formula of general Dedekind sums, (Chinese) Acta Math. Sinica 44 (2001), no. 1, 85-94.

[7] T. Zhang and W. Zhang, Hyper Cochrane sums and their hybrid mean value formula (in Chinese), Chinese Ann. Math. Ser. A 26 (2005), no. 4, 469-476.

[8] W. Zhang, On the general Dedekind sums and one kind identities of Dirichlet $L$-functions, Acta Math. Sinica 44 (2001), no. 2, 269-272.

[9] - On a Cochrane sum and its hybrid mean value formula, J. Math. Anal. Appl. 267 (2002), no. 1, 89-96.

[10] _ On a Cochrane sum and its hybrid mean value formula II, J. Math. Anal. Appl. 276 (2002), no. 1, 446-457.

[11] __ A sum analogous to Dedekind sums and its hybrid mean value formula, Acta Arith. 107 (2003), no. 1, 1-8.

[12] W. Zhang and H. Liu, A note on the Cochrane sum and its hybrid mean value formula, J. Math. Anal. Appl. 288 (2003), no. 2, 646-659.

Tianping Zhang and Wenpeng Zhang

Department of Mathematics

Northwest University

Xi'an, Shaanxi, P. R. China

and

Tianping Zhang

College of Mathematics and Information Science

Shannxi Normal University

Xi'an, Shaanxi, P. R. China

E-mail: tpzhang@snnu.edu.cn

wpzhang@nwu.edu.cn 